\title{
Deserción y rezago académico en el programa de medicina de la Universidad del Rosario, Bogotá, Colombia
}

Dropout and Graduation Delay in Undergraduate Medical Students. Universidad del Rosario, Bogotá,

Colombia

Deserção e atraso acadêmico no curso de medicina da Universidad del Rosario, Bogota, Colômbia

Andrés Isaza Restrepo MD Msc', Carolina Enríquez Guerrero Msc', Isabel Pérez-0lmos MD Msc ${ }^{3}$

Recibido: 09 de octubre de $2015 \bullet$ Aceptado: 15 de enero de 2016

Doi: dx.doi.org/10.12804/revsalud14.02.2016.08

Para citar este artículo: Isaza-Restrepo A, Enríquez-Guerrero C, Pérez-Olmos I. Deserción y rezago académico en el programa de medicina de la Universidad del Rosario, Bogotá, Colombia. Rev Cienc Salud. 2016;14(2):231-45. doi: dx.doi.org/10.12804/revsalud14.02.2016.08

\section{Resumen}

Introducción: el abandono y rezago en los programas de educación superior en Colombia y el mundo constituyen un problema de gran magnitud que afecta alrededor del $50 \%$ de estudiantes y conlleva un alto impacto personal, social y económico. Materiales y métodos: describir las características de la deserción estudiantil en el programa de medicina de la Universidad del Rosario y las variables asociadas. Se realizó un estudio descriptivo retrospectivo de las cohortes que ingresaron en los dos periodos académicos de 2003 y de la información académica de las cohortes de estudiantes registrados en el programa entre los años 2003 y 2008. Los datos se analizaron desde las perspectivas longitudinal y transversal. Resultados: la desvinculación definitiva total en las cohortes que ingresaron en los dos periodos académicos de 2003 fue de $47 \%$ y $36 \%$, la desvinculación temporal fue de $29 \%$ y $36 \%$, y obtuvieron el grado en el tiempo previsto el $22 \%$ y $19 \%$ de los estudiantes, respectivamente. La desvinculación promedio en cada periodo académico entre 2003 y 2008 fue 7,1\%. La principal explicación para la deserción fue de índole académica. Discusión: los porcentajes de deserción y el rezago académico en el currículo tradicional de medicina entre 2003 y 2008 fueron altos, similares a los de la educación superior en Colombia. Entre la complejidad que caracteriza este fenómeno, las estrategias pedagógicas y los aspectos académicos y curriculares parecen desempeñar un papel

1 Grupo de Investigación en Educación Médica y en Ciencias de la Salud de la Universidad del Rosario. Correo electrónico: andres. isaza@urosario.edu.co

2 Grupo de Investigación en Educación Médica y en Ciencias de la Salud de la Universidad del Rosario.

3 Centro de Investigación, Programa de Medicina, Universidad del Rosario. Grupo de Investigación en Educación Médica y en Ciencias de la Salud de la Universidad del Rosario. 
preponderante. Esta cohorte histórica y sus indicadores de deserción servirán de base de comparación para el nuevo currículo del programa de Medicina recientemente instaurado.

Palabras clave: educación médica, pregrado, deserción, rezago académico, currículo.

\begin{abstract}
Introduction: Dropout and graduation delay in higher education programs are a major problem worldwide, therefore also in Colombia, where it affects about $50 \%$ of the students, a fact that produces a high personal, social and economic impact. Objective: To describe the characteristics of the dropout and graduation delay of Medical School students at the El Rosario University, and its associated variables. Materials and methods: A retrospective, descriptive study of the two cohorts admitted in 2003 and of the academic information of students enrolled during the years 2003 through 2008 was performed. Data were analyzed from longitudinal and transversal perspectives. Results: Longitudinal analysis showed that both cohorts admitted in 2003 had a dropout rate of $47 \%$ and $36 \%$, graduation delay of $29 \%$ and $36 \%$; and $22 \%$ and $19 \%$ of the students, respectively, obtained their degree in the expected time. The mean dropout per academic period between 2003 and 2008 was $7.1 \%$. The main reazon for dropout was academic failure. Discussion: The dropout and graduation delay rates in the traditional curriculum of the Medical School between 2003 and 2008 were high, similar to those found in higher education in Colombia. Among the complexity that characterizes this phenomenon, teaching strategies and academic and curricular aspects seem to play a major role. This historical cohort and the associated dropout indicators will serve as a basis for comparison for the new curriculum of the Medical School program recently established.
\end{abstract}

Keywords: Medical education, Undergraduate, Student dropout, Graduation delay, Curriculum.

\title{
Resumo
}

Introdução: O abandono e atraso nos cursos de educação superior na Colômbia e no mundo constituem um problema de grande magnitude que afeta cerca do $50 \%$ de estudantes e leva um alto impacto pessoal, social e económico. Materiais e métodos: Descrever as características da deserção estudantil no curso de medicina da Universidad del Rosario e as variáveis associadas ao mesmo. Se realizou um estudo descritivo retrospectivo das coortes que ingressaram nos dois períodos acadêmicos do 2003 e da informação acadêmica das coortes de estudantes registrados no curso entre os anos 2003 e 2008. Os dados se analisaram desde as perspectivas longitudinal e transversal. Resultados: A desvinculação definitiva total nas coortes que ingressaram nos dois períodos acadêmicos de 2003 foi de 47 \% e 36 \%, a desvinculação temporal foi de $29 \%$ e 36 \%, e obtiveram o grau no tempo previsto o $22 \%$ e $19 \%$ dos estudantes respetivamente. A desvinculação média em cada período acadêmico entre 2003 e 2008 foi 7,1 \%. A principal explicação para a deserção foi de índole acadêmica. Discussão: as percentagens de deserção e o atraso acadêmico no currículo tradicional de medicina entre 2003 e 2008 foram altos, similares aos da educação superior na Colômbia. Entre a complexidade que caracteriza este fenômeno, as estratégias pedagógicas e os aspetos acadêmicos e curriculares parecem desempenhar um papel preponderante. Esta coorte 
histórica e seus indicadores de deserção servirão de base de comparação para o novo currículo do programa de Medicina recentemente instaurado.

Palavras-chave: Educação médica, graduação, deserção, atraso acadêmico, currículo.

\section{Introducción}

Uno de los problemas de mayor preocupación para el sistema educativo, dada su magnitud y las implicaciones económicas, sociales y personales que conlleva, es la deserción estudiantil. Esta expresión describe "el abandono prematuro de un programa de estudios antes de alcanzar el título o grado, durante un tiempo suficientemente largo como para descartar la posibilidad de que el estudiante se reincorpore" (1). El fenómeno se presenta en todos los niveles educativos y en los sistemas de educación pública y privada. Sin embargo, a pesar de las múltiples investigaciones que describen y develan sus causas, y de las intervenciones propuestas para controlarlo, no se logra revertir esta tendencia $(2,3)$.

Según el Sistema para la Prevención de la Deserción de la Educación Superior del Ministerio de Educación en Colombia (SPADIEs), la magnitud del problema tiende a un aumento progresivo tanto en Colombia como en países comparables. En el año 2013, la deserción universitaria en Colombia fue de 44,9\%.Además, la tasa anual de deserción en educación superior en Colombia (10,4 \% ) se ubicó en una posición intermedia entre la del Reino Unido $(8,6 \%)$ y las de Brasil y Estados Unidos (18\%). La tasa de deserción por cohorte en Colombia es de 45,3\%, mientras en México y la Argentina se aproxima al $43 \%$, en Venezuela y Chile al $53 \%$ y en Costa Rica llega hasta el $62 \%$; es decir, cerca de la mitad de los estudiantes que ingresan al sistema de educación superior desertan. Es particularmente interesante el dato de que el $75 \%$ de los casos de deserción ocurren durante los primeros cuatro semestres de la carrera (4).
En el marco del proceso de reacreditación de la calidad del programa de medicina de la Universidad del Rosario, adelantado en el año 2009, se encontró un aumento del porcentaje de deserción de $20 \%$ en 1999, a $26 \%$ en 2002. Por esta razón, el Comité de Acreditación de la facultad solicitó actualizar la información y avanzar en el estudio del problema. Para ello, se constituyó un grupo con interés en el estudio del tema y se diseñó un proyecto con el propósito de adoptar definiciones alrededor de la terminología relacionada con este problema, cuantificar su magnitud, describir sus características dentro del programa de medicina, analizar posibles variables asociadas con este y proponer planes de acción para su contención. Es pertinente anotar que para entonces no se contaba con procesos sistematizados de información sobre las características de la vida académica de los estudiantes como lo están en la actualidad.

La revisión de la literatura sobre el tema de la deserción en los programas de medicina recomienda avanzar en la investigación del fenómeno y su causalidad para producir conocimiento que permita diseñar medidas preventivas (5).

En este artículo se presentan los resultados de la primera aproximación sistemática al estudio de la deserción en el programa de medicina de la Universidad del Rosario, con datos recolectados en las cohortes de alumnos inscritos entre el primer semestre de 2003 y el segundo semestre de 2008.

Los autores consideran importante la publicación de estos resultados como base histórica 
para una comparación posterior en el contexto de la reforma curricular profunda adelantada en el programa de medicina, cuya primera cohorte cursa el quinto periodo académico en el segundo semestre de 2015.

\section{Materiales y métodos}

Para este estudio se adoptaron las definiciones de desvinculación definitiva (deserción) y desvinculación temporal (rezago) propuestas en la publicación de la Universidad Nacional de Colombia de 2007, por su precisión taxonómica (6). Las diferentes categorías fueron adaptadas a lo estipulado en los reglamentos académicos de pregrado de la facultad de medicina de la Universidad del Rosario, vigentes en el momento de la realización del estudio (Reglamentos Académicos 2002, Universidad del Rosario, Decreto Rectoral $N^{\circ} 716$ del de enero de 2002 y 2004, Decreto rectoral N 826 del 5 de octubre de 2004). Estas definiciones se describen a continuación.

Desvinculación temporal: es aquella que se da hasta por un máximo de dos periodos académicos consecutivos, prorrogable por un periodo, que puede explicarse por (a) razones académicas, cuya única justificación es el incumplimiento del requisito de segundo idioma; (b) sanción disciplinaria por dos periodos académicos consecutivos por faltas consideradas graves o gravísimas y (c) razones no académicas (económicas, enfermedades, estudios especiales, intercambios estudiantiles, viajes o desplazamiento por razones de seguridad, entre otras).

Desvinculación definitiva: es aquella que se da por más de dos periodos académicos y se denomina deserción en la Universidad del Rosario. Puede explicarse por (a) razones académicas, en cuyo caso se hablaba de fracaso académico en la Universidad del Rosario: reprobar por tercera vez una misma asignatura, obtener en un periodo académico un promedio inferior a tres punto cero
(3.0), no obtener el promedio exigido durante un periodo de prueba, perder tres o más asignaturas en un periodo académico, perder tres o más asignaturas dentro del ciclo de ciencias básicas, reprobar dos asignaturas básicas en un mismo periodo académico y la no aprobación por parte del Consejo Académico de una solicitud de repetición; (b) sanción disciplinaria, que consiste en la cancelación definitiva de la matrícula o expulsión, y (c) razones no académicas, cuando el estudiante no se matricula dentro del tiempo estipulado y no notifica por escrito el retiro del periodo. En la Universidad se denomina abandono del programa.

Rezago: el término rezago se aplica en el contexto del análisis longitudinal de una cohorte para indicar que el número de semestres o periodos académicos requeridos para obtener el grado fue mayor al previsto institucionalmente (doce semestres consecutivos en el programa de medicina de la Universidad del Rosario). Puede darse por razones académicas al reprobar asignaturas que deben ser repetidas o por razones no académicas.

Reintegro por estudios profesionales o fortalecimiento académico: están dirigidos a la contención del problema en la Universidad del Rosario y corresponden al caso de estudiantes que perdieron el cupo por razones académicas y se reintegran al programa de medicina en calidad de nuevos estudiantes tras haber aprobado programas especiales de apoyo académico que la Universidad del Rosario ofrece (Estudios Profesionales conocidos antes como "premédico" y Programa de Fortalecimiento Académico en Medicina). Antes del establecimiento del Programa de Fortalecimiento Académico en Medicina, los estudiantes que perdían varias asignaturas, sin perder el semestre, tenían la opción de realizar un periodo académico de "estudios premédicos profesionales" y si lo aprobaban, podían ingresar de nuevo a primer semestre como estudiantes de la Facultad de Medicina 
Diseño del estudio: para el análisis de la deserción en el Programa de Medicina de la Universidad del Rosario se realizó un estudio descriptivo retrospectivo con los datos de las dos cohortes que ingresaron en los dos periodos académicos del 2003 (2003-1 y 2003-2), y con la información académica de los estudiantes registrados en el programa entre los años 2003 y 2008. Estos datos se analizaron desde las perspectivas longitudinal y transversal.

Plan de análisis: para el análisis longitudinal se seleccionó el historial académico de los estudiantes de medicina de las cohortes que ingresaron en los dos periodos académicos del año 2003, cohortes a las que les correspondía obtener su grado en diciembre de 2008 y junio de 2009, los dos periodos más cercanos en el tiempo a la realización de este estudio. Se calcularon las frecuencias absolutas y relativas de estudiantes que obtuvieron su grado en el término previsto, el rezago académico y las desvinculaciones temporales y definitivas. Igualmente, se identificaron factores relacionados con la desvinculación y el rezago en las dos cohortes.

Para el análisis transversal se incluyó el historial académico de todas las cohortes de estudiantes, discriminadas por periodo académico cursado, entre el primer periodo del 2003 y el segundo periodo del 2008. Se calcularon las frecuencias absolutas y relativas de las desvinculaciones temporales y definitivas y los factores causales en cada una de ellas a partir del momento en que hubo registro institucional de esa información (2007-1). Se analizaron los ingresos por los programas de Fortalecimiento Académico y de Estudios Profesionales del Programa de Medicina de la Universidad del Rosario.

Se determinó también el porcentaje de reprobación en cada asignatura en los casos de desvinculación temporal o definitiva por causas académicas.
Por tratarse de una investigación documental institucional, se consideró como una investigación sin riesgo. Durante el proceso, se preservó la confidencialidad de la identidad e información personal de los alumnos inscritos en las cohortes de estudio

\section{Resultados}

Análisis longitudinal: se analizaron los registros académicos de 175 estudiantes distribuidos en las dos cohortes que ingresaron al programa de medicina en 2003. En la figura 1 se presenta el desenlace académico de los estudiantes que ingresaron a la primera cohorte de 2003 $(\mathrm{n}=87)$ en términos de graduación a tiempo, rezago por desvinculación temporal y desvinculación definitiva al finalizar el segundo semestre académico de 2008, periodo previsto para la obtención del grado sin rezago.

Las razones para la desvinculación definitiva de 41 alumnos en esta primera cohorte de 2003 fueron académicas en el $76 \%(\mathrm{n}=31)$, personales en el $2 \%(n=1)$, y no se lograron identificar en el $22 \%(n=9)$. Las razones para la desvinculación temporal fueron pérdida de asignaturas en el $68 \%(n=17)$, viaje para estudio de idiomas en $12 \%(n=3)$, otros estudios (Kaplan) $12 \%(n=3)$, sanción disciplinaria en $4 \%(\mathrm{n}=1)$ y otros motivos personales $4 \%$ $(\mathrm{n}=1)$. Las asignaturas que generaron desvinculación definitiva en esta cohorte fueron Biología Celular y Molecular, Bioquímica Estructural, Bioquímica Metabólica, Morfología Humana I, Neurociencias y Fisiología Humana (no se precisan números por asignatura, ya que con frecuencia los alumnos perdieron más de una asignatura). Estas asignaturas generaron desvinculación definitiva por razones académicas reglamentarias detalladas en su definición. Las asignaturas que generaron rezago fueron Farmacología Clínica $(n=8)$, Patología $(n=4)$, 

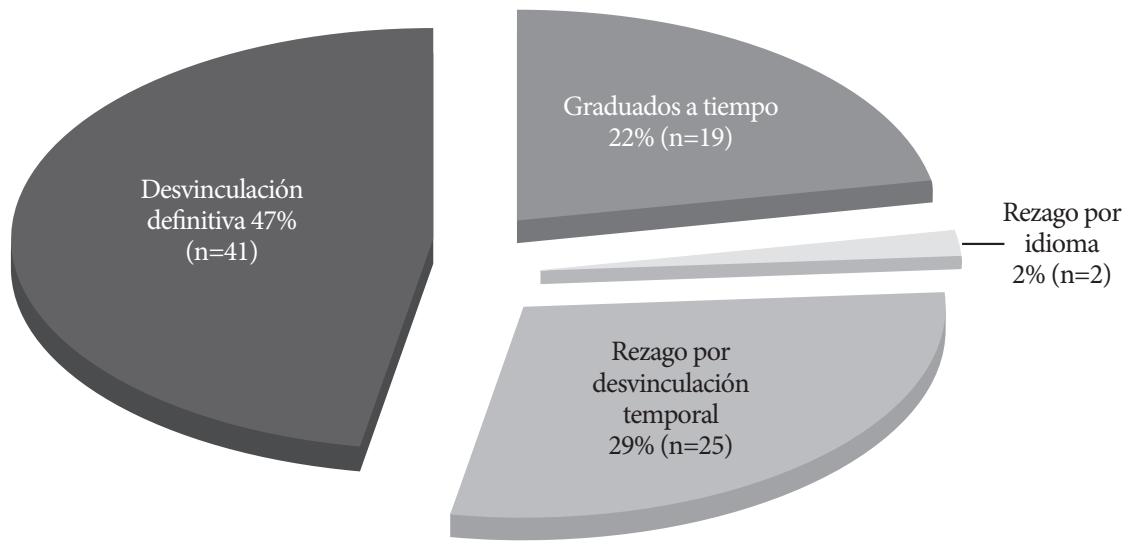

Figura 1. Desenlace cohorte de ingreso 2003-.. Programa de Medicina, Universidad del Rosario, 2003-2008 Nota: Cohorte de ingreso en enero 2003, grado en diciembre 2008 ( $n=87)$

Bioquímica Estructural ( $\mathrm{n}=3$ ), Biología Celular y Molecular ( $\mathrm{n}=2)$.

En la figura 2 se presenta el desenlace académico de los estudiantes que ingresaron en la segunda cohorte de 2003 ( $\mathrm{n}=88$ ), en términos de graduación a tiempo, desvinculación y rezago al finalizar el primer periodo académico de 2009, periodo previsto para la obtención del grado sin rezago.

En esta cohorte, las razones para la desvinculación definitiva fueron académicas en el $96,8 \%(\mathrm{n}=31)$, y las razones para la desvinculación temporal fueron pérdida de asignaturas en el 84,3\% ( $n=27)$, económicas en el 6,25\%

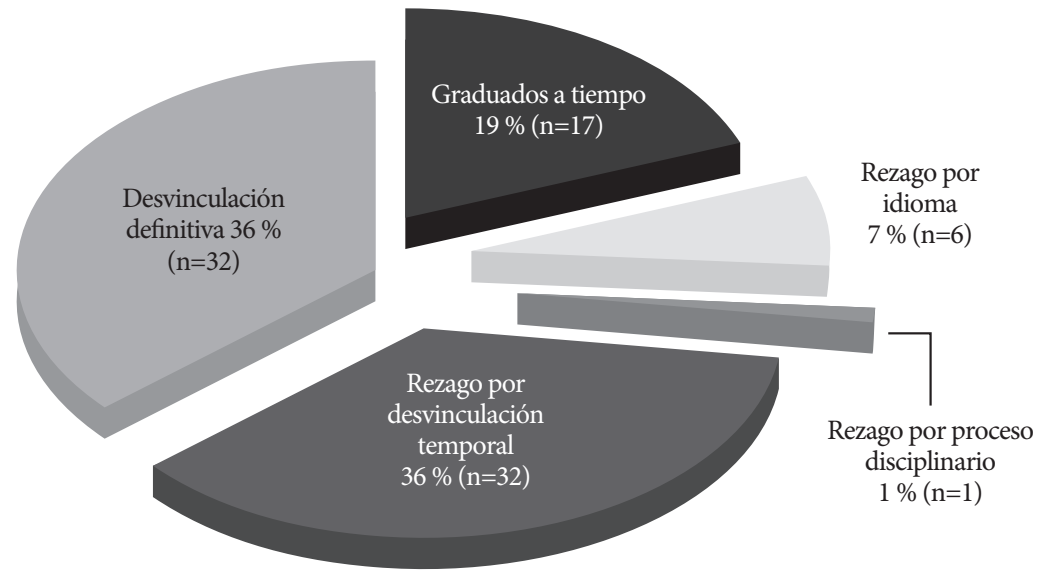

Figura 2. Desenlace cohorte de ingreso 2003-‥ Programa de Medicina, Universidad del Rosario, enero 2003-2009 Nota: Cohorte de ingreso en julio 2003, grado en junio de 2009 ( $n=88$ ). 


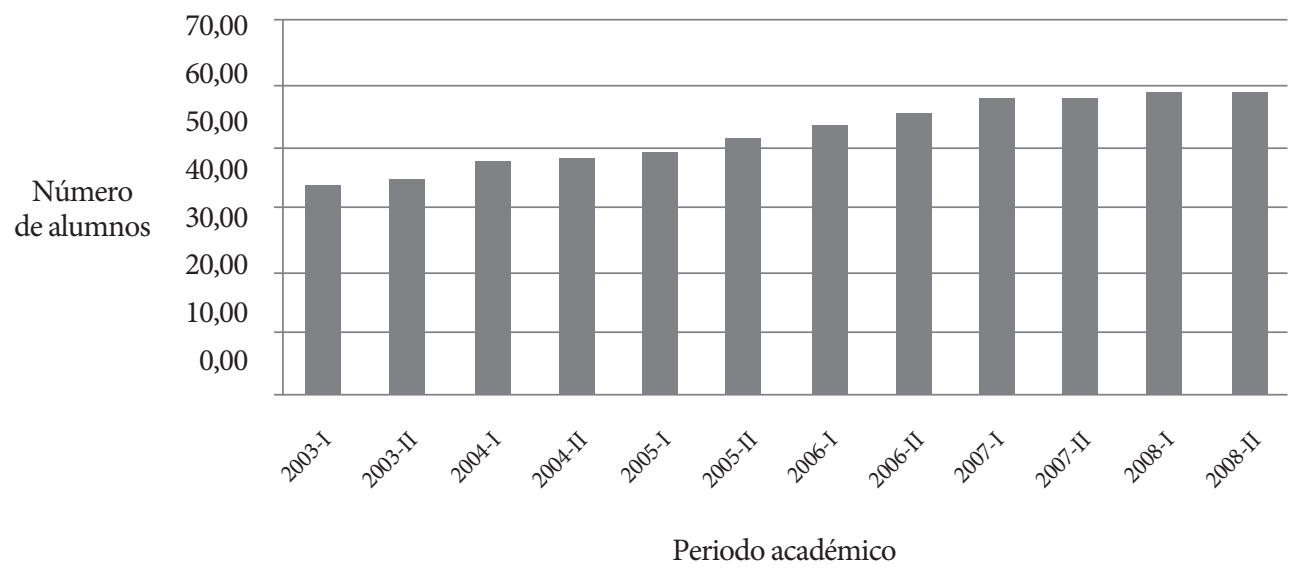

Figura 3. Número de alumnos en cada periodo académico, Programa de Medicina, Universidad del Rosario, enero 2003 diciembre 2008

( $\mathrm{n}=2$ ), o por otros estudios (Kaplan) 9,4\% $(\mathrm{n}=3)$. Las asignaturas que generaron desvinculación definitiva en esta cohorte fueron las mismas que para la cohorte previa, y las que generaron rezago fueron Farmacología Clínica $(n=12)$, Patología ( $n=6)$, Morfología Humana I $(\mathrm{n}=3)$, Biología Celular y Molecular $(\mathrm{n}=2)$, Bioquímica Estructural ( $\mathrm{n}=2)$, Morfología Humana II ( $\mathrm{n}=1)$ Medicina Tropical $(\mathrm{n}=1)$.

Análisis transversal: como marco de referencia, la figura 3 muestra la progresión del número de estudiantes matriculados en el programa de medicina durante el periodo analizado, que pasa de 677 alumnos en 2003-I a 976 en 2008-II, lo que representa un aumento del $30,6 \%$.

La tabla 1 presenta, en números absolutos y porcentuales, los casos de desvinculación temporal y definitiva por periodo académico, entre el año 2003 y el 2008. Respecto a la discriminación de las razones que explican las desvinculaciones según la información disponible, más adelante se describen algunos motivos registrados. Solo se cuenta con seguridad con los motivos relacionados con la exigencia del segundo idioma a partir del primer semestre de 2007.
En la tabla 1 también se presentan los ingresos a partir de los programas de Estudios Profesionales (premédico) y los reintegros por el Programa de Fortalecimiento Académico, estrategia dirigida hacia la contención del problema de la deserción en estudio desde el primer semestre de 2003.

La figura 4 muestra la tendencia creciente en el periodo de estudio entre 2003-1 y 2008-2 de los porcentajes de desvinculaciones temporales y desvinculaciones definitivas descritos en la tabla 1.

La tabla 2 presenta el porcentaje de pérdida de asignaturas por periodo académico entre el primer periodo de 2003 y el segundo periodo de 2008. Se observa que en la serie histórica se mantiene una tendencia a la pérdida de determinadas asignaturas por los estudiantes y constituye la causa más importante de desvinculación definitiva y de rezago.

\section{Discusión}

El presente estudio permitió determinar la magnitud de la deserción estudiantil en el programa de medicina de la Universidad del Rosario 
|saza-Restrepo A, Enríquez-Guerrero C, Pérez-Olmos |

Tabla 1. Número de alumnos inscritos, en desvinculación, y en programas especiales. Programa de Medicina, Universidad del Rosario, enero 2003 - diciembre 2008

\begin{tabular}{|c|c|c|c|c|c|c|c|c|c|c|c|c|c|}
\hline $\begin{array}{c}\text { Periodo académico / } \\
\text { descripción }\end{array}$ & 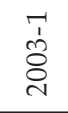 & 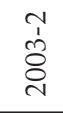 & $\begin{array}{l}7 \\
\dot{1} \\
8 \\
0\end{array}$ & 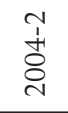 & $\begin{array}{l}71 \\
\text { ம் } \\
\text { 仓े } \\
\text { ก }\end{array}$ & 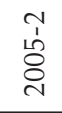 & $\begin{array}{l}71 \\
1 \\
0 \\
0 \\
0\end{array}$ & $\begin{array}{l}\tilde{1} \\
\vdots \\
\delta \\
\check{2}\end{array}$ & 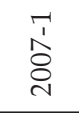 & $\begin{array}{l}1 \\
1 \\
0 \\
0\end{array}$ & 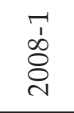 & 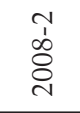 & total \\
\hline $\begin{array}{l}\text { Alumnos inscritos } \\
\text { programa medicina }\end{array}$ & 677 & 697 & 756 & 758 & 781 & 827 & 864 & 906 & 953 & 957 & 976 & 976 & 10128 \\
\hline Desvinculación Temporal & 19 & 12 & 16 & 10 & 13 & 11 & 16 & 16 & 36 & 42 & 49 & 24 & 264 \\
\hline Desvinculación definitiva & 26 & 27 & 18 & 16 & 20 & 19 & 33 & 38 & 66 & 59 & 55 & 83 & 460 \\
\hline Total desvinculación & 45 & 39 & 34 & 26 & 33 & 30 & 49 & 54 & 102 & 101 & 104 & 107 & 724 \\
\hline \% Desvinculación total & 6,6 & 5,6 & 4,5 & 3,4 & 4,2 & 3,6 & 5,7 & 6,0 & 10,7 & 10,6 & 10,7 & 11,0 & 7,1 \\
\hline Ingresos por premédico & 37 & 18 & 17 & 9 & 13 & 8 & 19 & 8 & 14 & 6 & 17 & 7 & 173 \\
\hline $\begin{array}{l}\text { Reintegros por } \\
\text { fortalecimiento } \\
\text { académico }\end{array}$ & 4 & 0 & 1 & 0 & 0 & 0 & 3 & 5 & 7 & 5 & 3 & 8 & 36 \\
\hline $\begin{array}{l}\% \text { ingreso programas } \\
\text { especiales }\end{array}$ & 6,1 & 2,6 & 2,4 & 1,2 & 1,7 & 1,0 & 2,5 & 1,4 & 2,2 & 1,1 & 2,0 & 1,5 & 2,1 \\
\hline
\end{tabular}

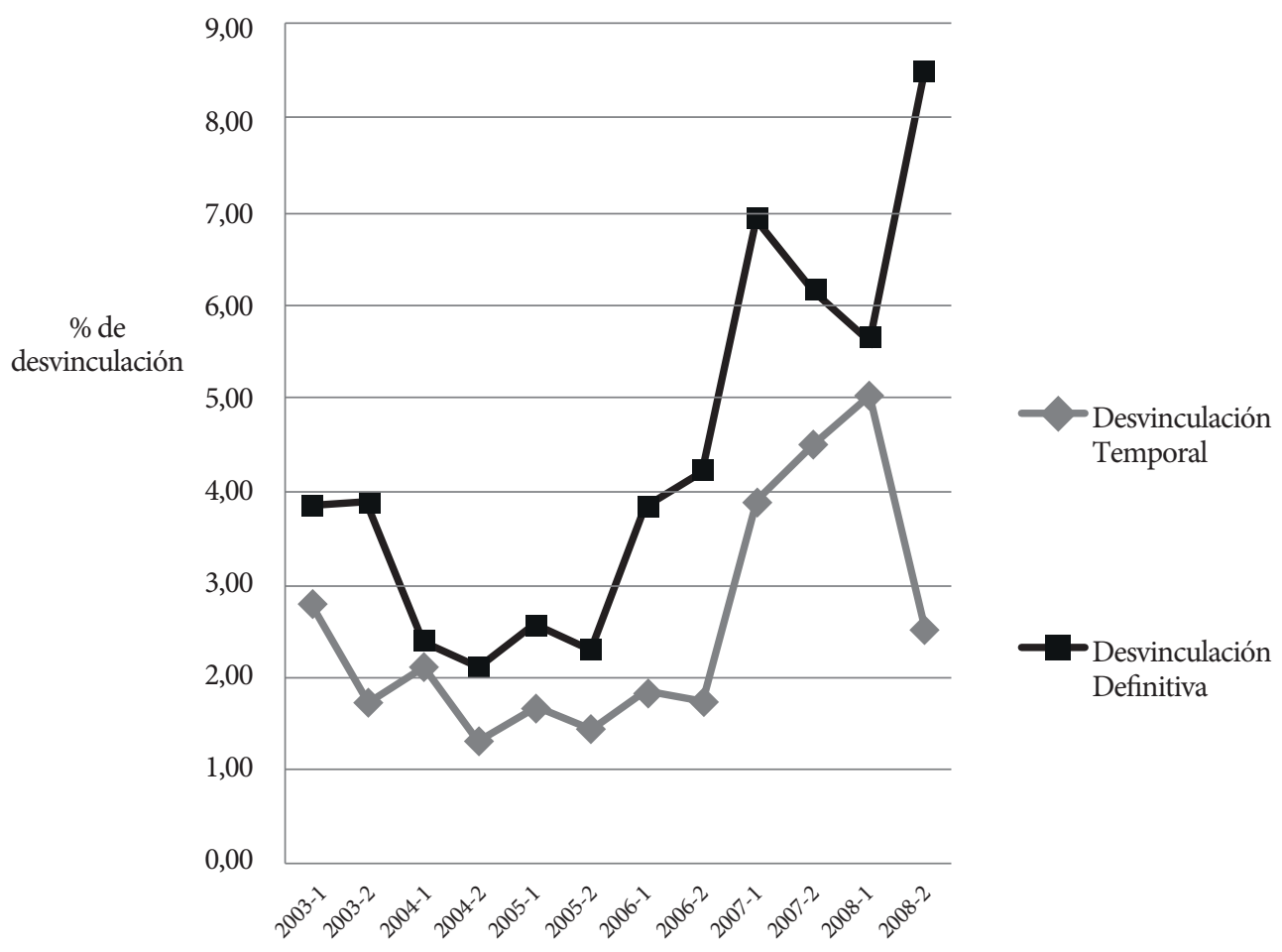

Figura 4. Desvinculación temporal y definitiva, tendencia. Programa de Medicina, Universidad del Rosario, Bogotá, enero 2003 - diciembre 2008 
Tabla 2. Porcentaje de alumnos con pérdida de asignaturas por periodo académico. Programa de medicina, Universidad del Rosario, enero 2003 - diciembre 2008

\begin{tabular}{|c|c|c|c|c|c|c|c|c|c|c|c|c|c|}
\hline Asignatura & 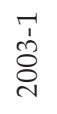 & 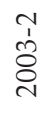 & 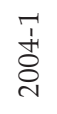 & $\begin{array}{l}N \\
\text { İ } \\
\text { O }\end{array}$ & 굴 & $\begin{array}{l}\text { N } \\
\text { மூ } \\
\stackrel{2}{1}\end{array}$ & 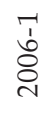 & 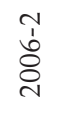 & $\begin{array}{l}\text { '. } \\
\hat{\delta} \\
\text { ते }\end{array}$ & $\begin{array}{l}\text { T⿱ } \\
\hat{\delta} \\
8\end{array}$ & $\begin{array}{l}7 \\
1 \\
0 \\
0 \\
0\end{array}$ & $\begin{array}{l}\text { 1 } \\
1 \\
0 \\
0 \\
0\end{array}$ & Promedio \\
\hline Bioquímica I & 27 & 27 & 29 & 6 & 3 & 24 & 29 & 25 & 37 & 30 & 12 & 35 & 24 \\
\hline $\begin{array}{l}\text { Morfología } \\
\text { humana I }\end{array}$ & 13 & 21 & 9 & 9 & 20 & 19 & 16 & 19 & 20 & 20 & 25 & 32 & 18 \\
\hline Bioquímica II & 22 & 25 & 20 & 2 & 4 & 14 & 16 & 17 & 16 & 20 & 9 & 28 & 16 \\
\hline Neurociencia & 8 & 20 & 12 & 11 & 14 & 11 & 15 & 10 & 15 & 8 & 21 & 27 & 14 \\
\hline Patología & 22 & 16 & 3 & 6 & 10 & 5 & 6 & 24 & 29 & 17 & 16 & 18 & 14 \\
\hline $\begin{array}{l}\text { Biología } \\
\text { celular y } \\
\text { molecular }\end{array}$ & 18 & 9 & 14 & 11 & 8 & 9 & 9 & 14 & 18 & 11 & 9 & 13 & 12 \\
\hline $\begin{array}{l}\text { Farmacología } \\
\text { clínica }\end{array}$ & 11 & 6 & 31 & 16 & 6 & 12 & 19 & 2 & 24 & 0 & 10 & 0 & 11 \\
\hline $\begin{array}{l}\text { Fisiología } \\
\text { humana }\end{array}$ & 5 & 4 & 3 & 10 & 12 & 11 & 16 & 19 & 12 & 2 & 1 & 16 & 9 \\
\hline Genética & 7 & 0 & 0 & 0 & 0 & 1 & 2 & 13 & 4 & 8 & 20 & 26 & 7 \\
\hline $\begin{array}{l}\text { Morfología } \\
\text { humana II }\end{array}$ & 9 & 1 & 5 & 2 & 3 & 4 & 5 & 4 & 3 & 11 & 8 & 6 & 5 \\
\hline $\begin{array}{l}\text { Farmacología } \\
\text { básica }\end{array}$ & 0 & 0 & 19 & 0 & 3 & 0 & 0 & 0 & 0 & 38 & 0 & 0 & 5 \\
\hline $\begin{array}{l}\text { Medicina } \\
\text { tropical y } \\
\text { enfermedades } \\
\text { infecciosas }\end{array}$ & 9 & 1 & 2 & 2 & 2 & 4 & 3 & 9 & 11 & 7 & 3 & 7 & 5 \\
\hline Inmunología & 6 & 6 & 10 & 3 & 6 & 5 & 3 & 1 & 4 & 9 & 0 & 1 & 5 \\
\hline Bioquímica III & 8 & 3 & 9 & 0 & 0 & 1 & 2 & 4 & 0 & 2 & 0 & 4 & 3 \\
\hline
\end{tabular}

e identificar factores relacionados con ella. Durante la vigencia del currículo tradicional evaluado entre el 2003 y el 2008, el índice de deserción mantuvo una tendencia creciente y alcanzó niveles preocupantemente altos.

Los porcentajes de desvinculación definitiva en las dos cohortes de ingreso al programa de medicina en 2003 en términos comparativos se encontraron dentro del rango de las tasas de deserción universitaria del sector privado en Colombia, que para la cohorte que ingresó en el primer periodo del 2003 fue de $47 \%$, e iguales o inferiores a la deserción definitiva en las dos cohortes de 2003 del programa de medicina de la Universidad Nacional correspondientes a $68 \%$ y $47 \%$, respectivamente (6).

Los resultados de la deserción de las dos cohortes evaluadas fueron inferior y superior, respectivamente, a la tasa de deserción global en pregrados de ciencias de la salud de Colombia, que en el año 2009 fue $39 \%$ y en 2013 no superaba el $40 \%$, según los resultados del 
Sistema para la Prevención de la Deserción en las Instituciones de Educación Superior de Colombia (spadies), publicados entre el 2009 y el $2014(7,8)$.

En términos de tendencia, las tasas de deserción estudiantil en la educación superior en Colombia venían en aumento progresivo desde 1998 hasta 2003, cuando comenzó a disminuir, pasando de $48,4 \%$ en 2004 a 44,9\% en 2008, mientras que el promedio para América Latina en 2006 estaba en $55 \%$ (7).

Con respecto a la graduación de los estudiantes, este estudio encontró que tan solo el $22 \%$ y el $16 \%$ de los estudiantes de las dos cohortes evaluadas obtuvieron su grado en el tiempo previsto. Estos resultados tampoco difieren sustancialmente de los encontrados en la literatura. La información registrada más recientemente en el SPADIEs permite establecer una tasa de graduación del 16,8 \% en el undécimo semestre y de $32,2 \%$ en el decimocuarto semestre, lo que muestra un rezago de al menos un año en cerca de la tercera parte de los alumnos inscritos (9).

Los estudios sobre este tema coinciden en resaltar la complejidad que caracteriza la explicación de la deserción o la permanencia de los estudiantes en el sistema de educación superior. Según el énfasis que se haga sobre las variables relacionadas, el discurso explicativo puede tener un enfoque psicológico, al considerar aspectos como las características individuales y los atributos de los estudiantes, o enfoques sociológicos que se centran en las dificultades de integración de los estudiantes al entorno de la educación superior. También enfoques económicos y organizacionales o, finalmente, enfoques integrados que explican la deserción a partir de las características de la institución, de los servicios que ofrece a los estudiantes, y conceden especial relevancia a la calidad de la docencia y a la experiencia en el aula $(8,10)$.
En la evaluación del problema en Chile, Díaz lo explica como un problema de motivación, derivado del logro de la integración académica y social, en la que intervienen factores como las características académicas preuniversitarias, institucionales, familiares, individuales o las expectativas laborales. Sin embargo, hay coincidencia general en que los factores académicos representan la primera causa de deserción, y así lo muestran nuestros resultados y otros estudios sobre este fenómeno en Colombia $(4,7,10)$.

En nuestro estudio, el factor académico fue la principal explicación de las desvinculaciones temporales y definitivas en ambas cohortes evaluadas, con porcentajes que oscilaron entre el 68 \% para explicar la desvinculación temporal entre los estudiantes de la primera cohorte de 2003, y el 96,8 \% para explicar la desvinculación definitiva en la segunda cohorte del mismo año. El peso de este factor es tan importante que las otras causas encontradas, y las descritas en la literatura sobre el tema parecieran secundarias.

En coincidencia con otros estudios, el periodo crítico en el cual el fenómeno de la deserción se presentó con mayor intensidad en las dos cohortes en estudio correspondió a los cuatro primeros semestres de la carrera, en los que se produjo el $74 \%$ de la deserción de estudiantes. El Ministerio de Educación Nacional menciona que más del $60 \%$ del total de deserciones ocurre en los primeros tres periodos académicos, y alrededor del $80 \%$ en la primera mitad de la carrera, lo que se denomina deserción temprana (7).

Para explicar por qué la deserción ocurre predominantemente en los primeros 3 a 4 periodos académicos, o más específicamente en la primera mitad de los estudios universitarios, los investigadores han señalado que es justamente en este periodo en el que se inicia el proceso de adaptación social y académica al medio universitario, en muchos casos en choque con el modelo 
pedagógico al que estaban expuestos en la educación secundaria y en gran diferencia con la homogeneidad sociocultural de los estudios del bachillerato. Se ha señalado que las razones para la deserción cambian según el periodo académico que se cursa, y si bien las razones académicas son las que explican la deserción temprana, este factor pierde peso en periodos más tardíos. Se plantea cómo algunos alumnos desarrollan una percepción negativa de su calidad de vida debido a factores como la elevada exigencia académica - particularmente en carreras como medicina e ingeniería-, la cultura universitaria, y las relaciones sociales que se dan en la universidad (11). Otros investigadores han demostrado que estos factores conllevan altos niveles de ansiedad, incluso aparición de trastornos emocionales derivados que cumplen un papel importante en la decisión de abandonar los estudios. De esta manera, un problema de bajo desempeño académico en los primeros semestres de estudios en medicina produce alteraciones que pueden derivar en trastornos emocionales de mayor severidad, estrés, ansiedad y depresión, constituyendo círculos viciosos de falla académica y trastorno emocional $(12,13)$.

$\mathrm{Al}$ analizar el fenómeno desde el punto de vista curricular, se encuentra que el inicio de la carrera corresponde, por lo general, a la transmisión de contenidos de asignaturas consideradas como las ciencias básicas de las diferentes disciplinas, estilo pedagógico que contribuye también a la falta de motivación de algunos estudiantes. Esta estructura es muy evidente en los currículos tradicionales de medicina. Así, los alumnos ingresan ilusionados a una carrera profesional con frecuencia motivados por un imaginario romántico de esta, se encuentran con estas asignaturas básicas que, en general, concuerdan poco con lo que será su desempeño profesional posterior, y al sumar estos aspectos con las estrategias pedagógicas magistrales, excesivamente tradicionales, poco estimulantes y con las deficiencias inherentes a los sistemas tradicionales de evaluación y el estrés adaptativo, se obtendrá un escenario decepcionante, exigente y punitivo, contexto propicio para la deserción $(14,15)$.

En este estudio se identificó que la principal causa de desvinculación temporal o rezago académico fue la pérdida de asignaturas, $68 \%$ y $84 \%$ en cada una de las dos cohortes analizadas. Se esperaría que las pérdidas en cada asignatura reflejaran falencias singulares de los estudiantes frente a unas metas de aprendizaje bien definidas en el currículo y que los porcentajes de pérdida de las asignaturas en el plan de estudios presentaran un patrón regular con el tiempo. Sin embargo, se observó que, aunque las asignaturas que pierden los estudiantes tienden a ser las mismas, los porcentajes de pérdida tienen un patrón irregular en las diferentes cohortes sin una explicación clara (tabla 2). Al intentar comprender la causa de esas variaciones en cohortes, los autores encontraron explicaciones diversas. Alguna norma dada al interior del programa para contener la deserción explicaría, por ejemplo, la disminución de los porcentajes de pérdida en Bioquímica I, II y III en el segundo periodo académico de 2004. En otros casos, se pudo concluir que las pérdidas dependieron de cambios en el sistema de evaluación empleado. En otros, la explicación se encontró en un aspecto que se podría denominar el "factor profesor", dado que el aumento o disminución de los porcentajes de pérdida coincidió con el cambio de los profesores responsables de la asignatura. También, en este estudio se encontró que la desvinculación por periodo académico, que osciló entre 2,1\% y $4,2 \%$ en los periodos comprendidos entre el 2003 y el 2006, pasó bruscamente a porcentajes entre 5,6\% y 8,5\% en los periodos del 2007 al 2008 (figura 4). Este aumento se explicó por la aplicación rigurosa de una norma del reglamento académico que exige a los estudiantes 
demostrar la suficiencia de inglés o un segundo idioma como requisito para el inicio de las ciencias clínicas a partir del $\mathrm{v}$ periodo académico del programa de medicina. Así pues, no parece suficiente reducir la explicación del problema a los bajos logros académicos de los estudiantes, lo que tampoco sería satisfactorio desde el punto de vista pedagógico, ni de la responsabilidad institucional sobre la formación de los estudiantes.

Cabe resaltar que en el periodo estudiado no hubo modificaciones en el sistema de selección de los aspirantes al programa de medicina, dado que varias investigaciones sugieren que parte de la explicación radica en ello. El estudio sobre la deserción en Colombia de 2013 citado encuentra que luego de los factores académicos, financieros, socioeconómicos e institucionales, están los de orientación vocacional y profesional, aspectos que se pretenden atender en algunos procesos de admisión (4). Las investigaciones evidencian que la selección de los estudiantes bajo criterios predeterminados y no al azar constituye un factor predictor de mejores logros académicos y contribuye a explicar la retención o la deserción en educación superior $(16,17)$. En este sentido, se ha relacionado la deserción universitaria con el grado de alfabetización académica, entendida esta como el nivel de desarrollo de competencias en lectura y escritura (18). La frecuente deficiencia en estas competencias en los estudiantes que ingresan a la educación superior en el medio genera serias dificultades para el aprendizaje. En un estudio sobre los factores que incidieron en el rendimiento académico de estudiantes de medicina de la Universidad del Rosario en el área de bioquímica, se encontró asociación positiva y estadísticamente significativa con los resultados de las pruebas de estado realizadas al final de la educación secundaria, el estatus de becario institucional y el ingreso directo a la carrera (sin estudios preuniversitarios previos) (19).
Aparte de las razones académicas, la exploración de causas para la desvinculación definitiva en estas cohortes permitió registrar algunos casos como "no identificados" o debidos a "razones personales". Al explorar estos motivos se lograron identificar viajes, algunos desplazamientos por razones de seguridad explicables en el contexto de la violencia de la época, y otros, probablemente por motivos económicos. Estos últimos explicaron con mayor exactitud la desvinculación temporal en 6,25\% de estudiantes de la segunda cohorte. Los estudios muestran que, además de que los factores socioeconómicos constituyen una importante barrera de acceso a la educación superior en América Latina, la permanencia o retención del estudiante en el sistema también depende de las oportunidades económicas para mantener el pago de la matrícula o el sostenimiento personal (7). Es probable que en este caso, por tratarse de una institución de educación superior privada que cuenta además con algunos programas de apoyo financiero, este factor no tuviera el mismo peso que en otras series en las que la segunda causa de la desvinculación fue la económica (4).

La información recolectada retrospectivamente para este estudio permitió una visión general de la deserción en el programa de medicina de la Universidad del Rosario, y puso en evidencia la falta de un seguimiento sistemático como el puesto en marcha para Colombia recientemente por el Sistema de Prevención de la Deserción en Educación Superior, que permita identificar y ponderar las variables asociadas con el fenómeno con mayor precisión, calcular el riesgo de deserción de cada estudiante a partir de condiciones académicas y socioeconómicas, y facilitar la selección, seguimiento y evaluación del impacto de estrategias de prevención y control de la deserción (7).

En este estudio no se contó con datos discriminados por sexo, edad, procedencia, carac- 
terísticas socioeconómicas, calificaciones preuniversitarias, características individuales de personalidad, prevalencia de trastornos emocionales, ausentismo o grado de aislamiento de los estudiantes desertores, factores que han sido señalados en otros estudios como asociados con el fenómeno, ni se puede aportar un análisis estadístico de las interrelaciones o el peso de estas variables en la determinación de la deserción $(8,12,20)$.

Tampoco se tiene información suficiente para identificar casos de migración de estudiantes al mismo programa en otras universidades, conocida como deserción institucional o el abandono definitivo del sistema educativo, por ejemplo, para vincularse al mundo laboral, o por razones familiares, o casos de migración hacia otros programas dentro de la misma institución, conocida como deserción interna o deserción de programa académico, desenlaces que ameritarían estudios posteriores con análisis independientes (7). El registro completo y detallado de la información obtenida en el seguimiento a los estudiantes es necesario para informar a las instituciones y para retroalimentar los procesos de selección de los estudiantes, el desarrollo de estrategias de enseñanza, currículo y los servicios de apoyo a los alumnos que lo requieran (20). Gracias al estudio y conceptualización del problema, en algunos ámbitos los modelos de seguimiento permiten identificar y clasificar a los estudiantes en riesgo de deserción, requisito indispensable para diseñar intervenciones dirigidas a la contención y solución del problema (21).

La deserción estudiantil es entonces un problema complejo, de gran magnitud, con ten- dencia a agravarse y con amplias repercusiones sociales, económicas, institucionales y personales que amerita atención permanente. Su contención constituye un imperativo ético para el sistema educativo y las instituciones. Por ello, el propósito del Ministerio de Educación es disminuir la tasa de deserción global en el país al $40 \%$ para 2010 , y al $25 \%$ en 2019 , según se plantea en el documento Visión Colombia II Centenario 2019 (22).

Los datos preliminares sobre la deserción de estudiantes que cursan el nuevo currículo del programa de medicina de la Universidad del Rosario muestran porcentajes inferiores al $3 \%$. Estos resultados dentro de un currículo actualizado según conceptos más progresistas en educación tienden a confirmar las reflexiones planteadas tras esta investigación, y su análisis detallado permitirá establecer un contraste con las tendencias históricas del problema presentadas en el presente estudio (23).

\section{Agradecimientos}

Al Dr. Carlos Moreno-Benavides del grupo de investigación NeURos, a la Dra. Ana Isabel Gómez, para entonces Directora del Programa de Medicina, miembros del Comité para el análisis de la deserción. Igualmente, a la Secretaría Académica de la Escuela de Medicina y Ciencias de la Salud de la Universidad del Rosario por su apoyo y contribuciones para la realización de este estudio.

\section{Descargos de responsabilidad}

Los autores declaran no tener conflictos de interés ni haber recibido financiación para la realización del estudio. 


\section{Referencias}

1. Himmel E. Modelos de análisis de la deserción estudiantil en la educación superior. Calidad en la educación. 2002;(17):91-108.

2. Universidad Nacional de Colombia \& ICFES. Estudio de la deserción estudiantil en la educación superior en Colombia. Documento sobre estado del arte. Convenio 107/2002 UN-ICFES2002. 2010.

3. Sánchez F, Quirós M, Reverón C, Rodríguez A. Equidad Social en el Acceso y Permanencia en la Universidad Pública. Determinantes y Factores Asociados. Bogotá: Centro de Estudios para el Desarrollo Económico (CEDE), Universidad de los Andes. 2002.

4. Ministerio de Educación de Colombia, spaDies. Deserción en Colombia 2013. Estadísticas 2013. Bogotá: SPADIES Sistema para la prevención de la deserción de la educación superior; 2013.

5. O'Neill LD, Wallstedt B, Eika B, Hartvigsen J. Factors associated with dropout in medical education: a literature review. Med Educ. 2011;45(5):440-54.

6. Universidad Nacional de Colombia. Cuestión de supervivencia. Graduación, deserción y rezago en la Universidad Nacional de Colombia. En: Dirección Nacional de Bienestar Universitario UNdC, 1. ${ }^{\text {a ed. }}$ Bogotá: Editorial Universidad Nacional de Colombia; 2007.

7. Guzmán C, Durán D, Franco J, Castaño E, Gallón S, Ministerio de Educación Nacional et al. Deserción estudiantil en la educación superior colombiana. Metodología de seguimiento, diagnóstico y elementos para su prevención. En: Nacional MdE, ed. 1. ${ }^{a}$ ed. Bogotá: Imprenta Nacional de Colombia; 2009.

8. Ministerio de Educación de Colombia, SPADIES, cede Facultad de Economía, Universidad de los Andes. Informe mensual sobre el soporte técnico y avance del contrato para garantizar la alimentación, consolidación, validación y uso de la información del spadies. Bogotá: Centro de Estudios sobre Desarrollo Económico, CEDE, Facultad de Economia, Universidad de los Andes; 2014.

9. Ministerio de Educación de Colombia, spadies, Sistema para la prevención de la deserción de la educación superior. Estadísticas de Deserción Estudiantil, Colombia, 2012. Bogotá: Ministerio de Educación de Colombia; 2012.

10. Díaz C. Modelo conceptual para la deserción estudiantil universitaria chilena. Estud Pedagóg. 2008;34(2):65-86.

11. Torres LE. Estado del Arte de la Retención de Estudiantes de la Educación Superior. Bogotá: Universidad Javeriana; 2010.

12. Han ER, Chung EK, Oh SA, Chay KO, Woo YJ. Medical students' failure experiences and their related factors. Korean J Med Educ. 2012;24(3):233-40.

13. Tamin SK. Relevance of mental health issues in university student dropouts. Occup Med (Lond). 2013;63(6):410-4.

14. Isaza A. Clases magistrales versus actividades participativas en el pregrado de medicina. De la teoría a la evidencia. Rev Est Soc 2005;(20):83-91.

15. Prince KJ, Van De Wiel M, Scherpbier AJ, Can Der Vleuten CP, Boshuizen HP. A Qualitative Analysis of the Transition from Theory to Practice in Undergraduate Training in a PBL-Medical School. Adv Health Sci Educ Theory Pract. 2000;5(2):105-16.

16. Urlings-Strop LC, Themmen AP, Stijnen T, Splinter TA. Selected medical students achieve better than lottery-admitted students during clerkships. Med Educ. 2011;45(10):1032-40.

17. O'Neill L, Hartvigsen J, Wallstedt B, Korsholm L, Eika B. Medical school dropout testing at admission versus selection by highest grades as predictors. Med Educ. 2011;45(11):1111-20. 
18. Olave-Arias G, Rojas-García I, Cisneros-Estupiñán M. Deserción universitaria y alfabetización académica. Educación y Educadores UniSabana. 2013;16(3):455-71.

19. Garzón R, Rojas MO, Riesgo L del, Pinzón M, Salamanca A. Factores que pueden influir en el rendimiento académico de estudiantes de Bioquímica que ingresan en el programa de Medicina de la Universidad del Rosario-Colombia. Educación Médica NLM. 2010;13(2):85-96.

20. Maher BM, Hynes H, Sweeney C, Khashan AS, O'Rourke M, Doran K, et al. Medical school attrition -beyond the statistics a ten year retrospective study. BMC Med Educ. 2013.

21. Arulampalam W, Naylor RA, Smith JP. Dropping out of medical school in the UK: explaining the changes over ten years. Med Educ. 2007;41(4):385-94.

22. Ministerio de Educación de Colombia. Deserción en Colombia, 2019. Bogotá: Mineducación. 2015.

23. Quintero G. Educación Médica. Diseño e implementación de un currículo basado en resultados de aprendizaje. 1. a ed. Bogotá: Editorial Universidad del Rosario; 2012. 